\title{
Product Differentiation for Software-as-a-Service Providers
}

Product differentiation of SaaS-based software bears potential for both customers and providers. We present a micro-economic based model to show the effects of product differentiation for this new type of software provisioning. By formalizing concepts of product differentiation for SaaS, this article shall establish basic understanding and form the foundation for further research. Furthermore, the model provides evidence that reproduction costs, which have mostly been neglected in literature related to quantitative software versioning so far, can have significant influence on both the optimal sales volume and service size in the context of SaaS.

DOI 10.1007/s12599-010-0142-4

\section{The Author \\ Dipl.-Ing. Dipl.-Wirt. Ing. \\ Arne Katzmarzik ( $\varangle$ ) \\ FIM Research Center Finance \& \\ Information Management \\ University of Augsburg \\ Universitätsstraße 12 \\ 86159 Augsburg \\ Germany \\ arne.katzmarzik@ \\ wiwi.uni-augsburg.de}

Received: 2010-05-01

Accepted: 2010-11-12

Accepted after two revisions by

Prof. Dr. Bichler.

Published online: 2011-01-13

This article is also available in German in print and via http://www. wirtschaftsinformatik.de: Katzmarzik A (2011) Produktdifferenzierung für Software-as-a-Service-Anbieter. WIRTSCHAFTSINFORMATIK. doi: 10. 1007/s11576-010-0258-7.

(c) Gabler Verlag 2011

\section{Introduction}

Progress in information technology (IT) such as the service-oriented architecture (SOA) paradigm and advances in web based communication facilitate new types of software provisioning such as web services (Papazoglou et al. 2007). Enabled by web services technologies, the service-oriented software provision- ing (SSP) model "Software-as-a-Service" (SaaS) allows to integrate standard software into online service infrastructure (Cheng et al. 2006, p. 521; Lehmann and Buxmann 2009). Customers may easily rent functionality and use this software via web clients instead of running licensed software on their own IT infrastructure. SaaS bears advantages for both providers and customers. SaaS providers can primarily profit from economies of scale by addressing more customers (Walsh 2003; Sääksjärvi et al. 2005). Advantages for customers are e.g. lower IT procurement costs or faster access to new technology, functionality and upgrades (Walsh 2003; Susarla et al. 2009, p. 207). Thus, this emerging market will attract more and more customers and has already reached a significant size of $\$ 9.6 \mathrm{bn}$, still shows two-digit annual growth rates and is predicted to increase to $\$ 16$ bn in 2013 (Gartner 2009). In particular providers can profit from this market development. However, they must diversify their offers to be attractive for both existing and new customers. The concept of product differentiation may lead to a win-win situation for both providers and customers. Therefore, a decision support model considering both customers' and providers' needs as well as the characteristics of SaaS is crucial.

The objective of this paper is to contribute to close this identified gap by developing a normative approach to support decisions from the perspective of a monopolistic SaaS provider. In the introduced model, a provider can maximize its return by changing the granularity of its offered services, i.e. it can spread the functionality on several smaller services instead of offering all in one monolithic service. Based on micro-economic theory and the information systems (IS) research stream of software versioning - or in the following just versioning our scientific contribution is two-fold: First, based on the ideas of versioning, we discuss if models from this stream can be applied to SaaS and lay a conceptual foundation for product differentiation for SaaS by introducing a model based on the demand curve which is extended by characteristics of SaaS. Second, we elaborate from literature that variable reproduction costs - in contrast to classic software provisioning (CSP) - cannot be neglected for SaaS and integrate these costs into our model, which has not been state of the art so far. To improve comprehensibility, the model is illustrated by studying a real world case with data from a major provider.

The remainder of this text is organized as follows: In Sect. 2, we give an overview of related literature. Additionally, this section lays the conceptual fundament for this paper (including the definition of important terms). In Sect. 3, the decision model is presented, analyzed and illustrated by an operationalization. After a discussion in Sect. 4 we conclude in Sect. 5.

\section{Product Differentiation of Software Goods}

Product differentiation can be divided into two dimensions, vertical and horizontal differentiation (Cremer and Thisse 
1991). Vertical differentiation refers to offering a good in multiple versions each differing in size and price (Bhargava and Choudhary 2001). Horizontal differentiation refers to offering goods separately in parallel independent versions to address customers who request only specific parts (Weber 2008). As SaaS includes characteristics of both dimensions as we shall see below, we provide an integrated view on both dimensions and generally speak of product differentiation. This concept has been applied in the stream of versioning for IS goods, and there mostly for CSP, i.e. when customers purchase software from a provider as installation package and are themselves responsible for running it (Phillips 2009). Thus, we start our examination with an overview of effects and literature on the application of product differentiation for CSP in Sect. 2.1. We discuss their applicability to SSP models and in particular SaaS in Sect. 2.2. We conclude this section with a review of related work and point out the need for further research on decision support in Sect. 2.3.

\subsection{Effects of and Literature on Product Differentiation}

Demanders of software functionality are heterogeneous in demand for functionality and willingness to pay (WTP) (Jing 2000). By differentiating software products, providers may try to adjust their software to be more customer specific and thus may address a broader target group to extend sales (Shapiro and Varian 1998). Due to the characteristics of standard software, customers usually do not require all the functionality included in an offer (Raghunathan 2000). As product differentiation enables purchasing only parts of the functionality instead of the whole, customers who would have purchased the whole if this were offered exclusively, but only have demand for parts of the functionality, can purchase solely the parts they actually require (Bhargava and Choudhary 2001). This may result in a decreasing total amount of sold functionality.

Pricing is another important aspect when being faced with customers of heterogeneous demand and WTP. Standard software usually contains parts of functionality which customers do not require, but customers are forced to buy these not required parts (Raghunathan 2000). Hence, the available budget has to be spent both on required and not required functionality. Customers, however, are only willing to pay for required functionality as using this generates added value. Product differentiation makes it possible to offer functionality in smaller parts instead of one holistic version. This ideally allows customers to obtain exactly the functionality requested, i.e. the one perfectly fitted to their specific demand (Choudhary et al. 2005). As the WTP of customers depends on their specific demand (Weber 2008), the total budget remains (nearly) unaffected if not requested functionality is excluded. Therefore, providers may attain higher prices per piece of functionality if an offer is more demand specific. Thus, providers must carefully trade off between the effects of attainable prices and acquisition of new customers against the possibility of selling in sum less functionality.

Product differentiation also has effects on costs. First, software products have to be made accessible for customers and thus providers have reproduction costs (Varian 1997). As product differentiation may positively affect the number of customers, reproduction costs usually increase. Furthermore, the total functionality has to be cut into smaller or parallel versions which means additional effort to split up functionality and offer new packages (Weber 2001). Therefore, a higher degree of product differentiation leads to increasing costs.

Literature on differentiating CSP products goes even beyond finding an optimal number of versions in the tension area of the mentioned basic effects. Examinations have been conducted in more specific contexts such as competition (Jones and Mendelson 2005; Wei and Nault 2006), licensing in software contracts (Zhang and Seidmann 2002), interorganizational systems (Nault 1997), free download policies (Cheng and Tang 2010), network externalities (Jing 2000), and fighting digital piracy (Chellappa and Shivendu 2005). In the following, we discuss if the basic effects can be simply transferred to SaaS.

\subsection{Special Characteristics of SaaS: Modeling Issues}

To elaborate differences between CSP and the SSP model SaaS, we must have a closer look at this uprising provisioning type: SaaS refers to a software provisioning model hosting a standard software application on an Internet accessible server (Lehmann and Buxmann 2009).
Thereby, customers rent an application from a provider on a per-use or perperiod basis, and the provider itself is responsible for delivering, securing and managing application, data and underlying infrastructure (Kaplan 2007). Thus, SaaS bundles software functionality with infrastructure services (Fan et al. 2009, p. 661). At this point, we have to clarify that here we are not examining the effects of splitting the bundle of functionality and infrastructure, instead we want to study effects of splitting functionality into its parts.

Valente and Mitra (2007) state that there are enormous differences in customer access to software functionality and responsibility of providers. Therefore, the presented models from the stream of versioning as well as the effects elaborated in versioning literature cannot simply be transferred to SaaS. We will discuss differences between both provisioning types below and elaborate modeling issues that have to be considered in a decision model.

Decision problem: In models for CSP, providers offer a flagship version containing the total functionality and also inferior versions which are created by removing functionality from the flagship version (Bhargava and Choudhary 2001), or they offer several smaller parallel versions as independent products which can be re-bundled to a flagship version (Weber 2008). In SSP models, functionality is offered as service. A (web) service is a software artifact containing certain business functionality (Papazoglou and van den Heuvel 2007). According to the SOA paradigm, services can be integrated into applications and/or re-combined to applications. If functionality is offered very granular, i.e. it is split up into many small services, customizability and flexibility increase as numerous "versions" are possible. In those terms, Anderson (2006) describes the ideal of a highly granular SaaS offer: Customers may select exactly the functionality they require and so customize their 'own service' by compiling it from all available artifacts. At this point, we have to mention that product differentiation for SaaS is situated both in the vertical and the horizontal dimension since splitting allows on the one hand several inferior versions with less functions or features, but also on the other hand parallel versions with disjoint functions or features. However, it still depends on the functionality characteristics if a product differentiation prob- 
lem belongs to only one or both dimensions. Thus, the service granularity (or just granularity), which refers to the size of a service, is a viable instrument for differentiation of SaaS products (Haesen et al. 2008, p. 383). This leads to Modeling Issue 1: Adjusting granularity has to be considered in a model for SaaS instead of finding a number of versions to ensure full flexibility in differentiating SaaS products.

Sales Volume: Product differentiation makes it possible to address additional customers that are only interested in parts of the offered functionality. In versioning literature, this effect with impact on sales volume, i.e. the amount of sold functionality, is handled in two ways. Models of the first type such as Bhargava and Choudhary (2001) are built on assumptions that the sales volume will increase as additional customers buy inferior versions and the sale of the flagship version is not affected. Models of the second type such as Nault (1997) consider that customers who would buy the flagship version might choose an inferior version instead. The models of the latter type argue that the number of customers increases, but it is not guaranteed that the overall sales volume increases as only few customers may buy the flagship version and many customers only inferior versions. Since highly granular SaaS offers allow very high customizability, assumptions of the second type of models are more realistic and should be adopted: Therefore, both positive and negative variation of the sales volume should be considered in a model (Modeling Issue 2).

Pricing: In versioning models, pricing is based on WTP and specific demand of customers (Weber 2008, p. 448). These models usually assume that additional value generated for a customer may decrease with every further piece of functionality (Ghose and Sundararajan 2005), since the amount of not required functionality increases with larger offers. As SaaS is also standard software as the examined software in versioning models, we postulate - referring to pricing aspects of versioning models (cp. 2.1) - that this assumption has to be adopted leading to Modeling Issue 3: The higher the granularity of SaaS offered functionality the more flexibly customers can select parts according to their specific demand. Customers can now directly spend their budget on requested functionality leading to higher attainable prices per piece of functionality.

Costs: Modifying products requires a technical effort that also has to be considered in a holistic economic view. In versioning models, costs are split into production and reproduction costs. Production costs on the one hand consist of costs for implementing functionality and maintenance (Banker et al. 1991), and on the other hand of costs for granularity: With increasing granularity, providers offer more services, i.e. functionality has to be cut into several modules, and also interfaces have to be provided. Interfaces must ensure that services can be accessed by customers or by other services which require their functionality (Krafzig et al. 2005). Furthermore, granular services must be composed in a way that they can work together in order to rebuild business processes (Arsanjani et al. 2008). Heinrich and Fridgen (2005) state that for $m$ services at least $m$, i.e. a single interface per service, but up to $\frac{m \cdot(m-1)}{2}$ interfaces, i.e. all services are connected with each other, have to be provided. Thus, more services make implementing interfaces and their composition more complex and costly (Haesen et al. 2008), resulting in Modeling Issue 4: Costs for granularity increase with granularity, whereas costs for implementing functionality and maintenance are mostly independent of granularity.

Reproduction costs arise for making functionality accessible for customers. For CSP, vendors usually provide a copy on a data transfer medium or a download server, these costs however being of insignificant size and usually neglected in decision models (Bhargava and Choudhary 2001; Varian 2000). In contrast, a SaaS provider is responsible for hosting and running computations. The more functionality a provider sells, the more computations have to be conducted and the higher call frequencies of services and data transfer are, resulting in costs for infrastructure (Boerner and Goeken 2009). This causes communication and computing costs which are of significant size for SaaS (Lehmann and Buxmann 2009) resulting in Modeling Issue 5: Reproduction costs must be considered in a model for SaaS.

Since varying the granularity of SaaS shows enormous differences in contrast to versioning for CSP, we concentrate on the identified modeling issues in the following. In summary, Table 1 compares CSP and SSP based on the discussion above and gives examples for practical application.

\subsection{Related Work on Product Differentiation of SSP and Granularity}

A lot of quantitative models for CSP stemming from the related research area of versioning exist, but cannot be applied to SaaS as discussed. With respect to the special characteristics of SaaS, Lehmann and Buxmann (2009) state that there is a need for new pricing models for SaaS and following decision support. Though most articles dealing with SaaS still concentrate on qualitative aspects such as Benlian (2009), Benlian et al. (2009) or Mietzner and Leymann (2008), there are a few quantitative decision models concerning SaaS and the highly related area of web services which we analyze below.

Most of the existing research of this upcoming research stream is about competition and factors for offering services successfully on the market. For SSP, Cheng et al. (2006) and Fan et al. (2009) have examined the effects of provisioning strategies. Cheng et al. (2006) analyze three different SSP strategies for providers and investigate under which conditions these strategies are profitable. Fan et al. (2009) examine short- and long-term competition between providers of SaaS and CSP. With a game theoretical approach, the authors find that SaaS providers have to face high introduction costs in the short run, but have advantages in the long run due to an increasing customer base. Both papers show the economic potential of SSP from a strategic perspective, but do no focus on details.

Another important success factor for SSP is the service level, i.e. the availability of offered functionality, as providers have to guarantee access to their functionality (Fan et al. 2009, p. 662). Zhang et al. (2009) examine effects on sales volume and pricing due to increasing service levels. Bhargava and Sun (2008) show how contingency pricing can be applied to IT services and find that customers are willing to pay higher prices dependent on the provided service level. The mentioned papers provide evidence how providers can positively affect prices, but do not consider the granularity of services.

Erl (2005, p. 557) outlines that adjusting granularity is an important eco- 
Table 1 Comparison between CSP and SSP based on the discussion

\begin{tabular}{|c|c|c|c|}
\hline & \multirow[t]{2}{*}{ CSP } & \multicolumn{2}{|l|}{ SSP } \\
\hline & & Web service & SaaS \\
\hline Definition & $\begin{array}{l}\text { Standard software offered as } \\
\text { installation package. Running and } \\
\text { hosting are independent of sales. }\end{array}$ & $\begin{array}{l}\text { Functionality that can be integrated } \\
\text { into applications according to the } \\
\text { SOA paradigm. It is offered on an } \\
\text { Internet accessible server. Running } \\
\text { and accessibility are ensured by the } \\
\text { provider. }\end{array}$ & $\begin{array}{l}\text { Provisioning strategy based on } \\
\text { web service technology: } \\
\text { Standard software (incl. } \\
\text { frontend) is run, hosted and } \\
\text { provided via Internet. }\end{array}$ \\
\hline Examples & Microsoft (MS) Office & $\begin{array}{l}\text { Query of credit rating provided by } \\
\text { rating agency }\end{array}$ & $\begin{array}{l}\text { SAP-CRM-On-Demand, } \\
\text { NetSuite ECOMMERCE } \\
\text { (NSE) }\end{array}$ \\
\hline $\begin{array}{l}\text { Implementation } \\
\text { of product } \\
\text { differentiation }\end{array}$ & $\begin{array}{l}\text { Versioning: Providers offer different } \\
\text { versions to acquire new customers } \\
\text { with a more demand specific offer. } \\
\text { Reproduction costs per customer are } \\
\text { neglectably small. }\end{array}$ & \multicolumn{2}{|c|}{$\begin{array}{l}\text { Granularity: Providers offer independent, but combinable services. With } \\
\text { higher granularity, i.e. smaller and more diverse services, customers may } \\
\text { select functionality fitting their specific demands, and thus more customers } \\
\text { are addressed. Costs for cutting functionalities, for interfaces and service } \\
\text { composition increase with higher granularity. Reproduction costs depend } \\
\text { on communication and computing and can be of significant size. }\end{array}$} \\
\hline $\begin{array}{l}\text { Examples of } \\
\text { production } \\
\text { differentiation }\end{array}$ & $\begin{array}{l}\text { Vertical: MS Office Professional, MS } \\
\text { Office home } \\
\text { Horizontal: MS Word, MS Excel }\end{array}$ & $\begin{array}{l}\text { Vertical: Query is based only on data } \\
\text { of few periods } \\
\text { Horizontal: Query delivers additional } \\
\text { information about subject }\end{array}$ & $\begin{array}{l}\text { Vertical: NSE with constrained } \\
\text { analysis functionality } \\
\text { Horizontal: NSE Web Shop, } \\
\text { NSE B2B }\end{array}$ \\
\hline
\end{tabular}

nomic factor for web services and SOA. In a more detailed research on granularity, Haesen et al. (2008) elaborate three dimensions of decisions on granularity. The functionality dimension is about reducing production costs due to a higher re-use share. The data dimension refers to reducing communication costs due to the amount of transfered data. Whereas the objective of both dimensions is reducing costs, the business value dimension is about increasing sales volume and addressing more clients. Holschke et al. (2009) consider granularity as a decision variable in the functionality dimension. They examine which granularity is cost minimizing in reconstructing an existing IT system. In the business value dimension, Lee et al. (2006) present a versioning approach for web services. They examine how sales volume, aspired quality of a flagship service and the total costs are affected by a free inferior version, but the authors do not consider effects of chargeable inferior services. These papers focus on sizing and granularity, but consider only isolated relevant aspects (cp. Modeling Issues) instead of taking on a holistic view.

Granularity of software has also been subject of research concerning the related area of components. Based on Parnas' (1972) work on modulization and Szysperski's (1998) analysis on component technologies, research was about finding a suitable size of software modules. Such approaches to granularity either have a functional focus (Albani et al. 2003), i.e. clustering similar functionalities, a technical focus (Kim and Chang 2004), i.e. how modules or services can be composed to make up an application, and an economic focus (Wang et al. 2005), which aims at finding a granularity in order to reduce costs in implementing systems. The economic approaches primarily focus on the cost dimension, but not the sales dimension.

In conclusion, we can state that there is a lack of quantitative research considering SaaS. Additionally, to the best of our knowledge, there is no publication that takes a holistic quantitative approach and examines the granularity of SaaS considering sales volume, pricing and technical aspects. Thus, we aim to fill this research gap by developing a model which takes into account the elaborated modeling issues.

\section{A Model Supporting Product Differentiation Decisions for SaaS Providers}

We now present a micro-economic model to support granularity decisions on SaaS products. We introduce the general form of our model and the underlying basic assumptions in Sect. 3.1. This is followed by a simplified model with assumptions concerning behavior of the market and production costs to show fundamental relationships in Sect. 3.2. Here, we present an analytical solution and operationalize our model with a real world case serving as running example in the following. In Sect. 3.3, we extend the simplified model by variable reproduction costs (cp. Modeling Issue 5).

\subsection{General Form of the Model}

\subsubsection{Basic Assumptions and Notation}

The theoretical fundament of the model is formed by the demand curve representing the relation between prices and sales volume (Varian 2009) as well as the identified Modeling Issues based on the literature review. To set up the demand curve, we have to make two assumptions:

Assumption 1 In our one-period model, a monopolistic SaaS provider offers functionality, namely the amount $F$ mea- 
sured in size units (SU). ${ }^{1}$ Originally, the functionality is implemented as coherent block and offered in one piece to $N$ customers. Each customer may either buy or not. In addition, there is a technical module required to run the functionality. This technical module is not included in $F$ and its price is included in using the functionality.

Thus, the maximum demand $Q$, i.e. the quantity of salable functionality, is the product of offered amount and maximum number of customers $(Q=F \cdot N)$. For reasons of simplicity, in the following we consider $Q$ instead of its calculation $(F \cdot N)$.

Assumption 2 The market for selling one single service comprising the whole functionality is given by a demand curve depending on the price $p$. The demand curve is continuous and linear, has a negative slope, is characterized by the maximum demand $Q$, and the market parameter $\beta$.

With both assumptions, the price for every possible demand $x$ from 0 to $Q$ can be calculated.

This relation is now enhanced by granularity aspects elaborated in the literature review. According to Modeling Issue 1, the provider also can offer the functionality in several more granular services to improve profits:

Assumption 3 The functionality can be cut into $M$ services of same size FS (= $\left.\frac{F}{M}\right)$. Services are not overlapping. The technical module can be used with any service combination without modification.

Based on this assumption, we can introduce the decision variable degree of granularity $g \in[0 ; 1[$ as normalization and measure of the functional size, whereby $g=0$ refers to no granularity and higher values of $g$ refer to more granular services.

As identified in Modeling Issue 2, offering not all SU in one, but in several more granular services may help acquire new customers ( $N$ increases). As services become smaller, this bears the risk that customers may only purchase services containing actually required functionality (less than all offered $F$ SU).
Assumption 4 The maximum demand may vary dependent on granularity.

With higher granularity the more flexible customers can select functionality which fits their specific demand better. Thus customers can more directly spend their budget on required functionality as they only want to pay for this. According to Modeling Issue 3, following relation between prices and granularity is assumed:

Assumption 5 The price per SU increases with higher granularity.

We now can introduce the function $h(g)$ representing the increase in price per SU subject to the degree of granularity. According to Assumptions 4 and 5, the provider can influence the market, namely the maximum demand and the attainable price, by modifying the granularity of its services.

These modifications have effects on the total costs (denoted by $C$ ) consisting of production and reproduction costs. Production costs consist of costs for implementing and maintaining functionality (denoted by $C_{p}$ ) and costs for granularity (denoted by $C_{g}(g)$ ), i.e. for splitting the functionality into more services, and for interfaces. To satisfy Modeling Issue 4, we assume:

Assumption 6 Implementation and maintenance costs are fixed. Costs for granularity increase with higher granularity.

To ensure Modeling Issue 5, we consider reproduction costs $C_{r}(x)$ due to higher communication and computing effort:

Assumption 7 Reproduction costs depend on the amount of sold functionality.

With these assumptions of market and costs, we can develop a model in which a provider can determine the optimal degree of granularity in order to maximize its return.

\subsubsection{Model Development}

In the first part of this subsection, we develop our optimization model for determining the optimal number of SU to sell.
In the second part, we integrate effects of granularity into the model.

The actual number of sold SU can be determined by the demand curve. According to mirco-economic theory, this figure can be calculated as difference between the maximum demand and the product of price per SU and market parameter $\beta$ measuring the impact of the price. Thus, prices and sales volume correlate negatively (Varian 2009):

$x(p)=Q-\beta \cdot p$

By inverting the calculation, the price can be written as a function of the demand:

$p(x)=\frac{Q-x}{\beta}$

Though it is more intuitive from an entrepreneur's point of view to set the price instead of setting demand, we take the latter perspective (2) as it is mathematically easier to process and the model development is easier to follow. The return can be calculated as a product of price and sold SU minus costs:

$R(x)=\frac{Q-x}{\beta} \cdot x-C$

Equation (3) can be employed to calculate the return if all functionality is offered in one single service, i.e. $F S=F$. Now we model the effects of granularity, i.e. $F S<F$. According to Assumption 3, we introduce the degree of granularity, which can be calculated in two ways: as a relation of the offer share, i.e. the size per service over the total amount of offered functionality, and alternatively depending on the number of services. To be more intuitive, $g=0$ shall refer to no (zero) granularity.

$$
\begin{aligned}
g & =g(F S)=1-\frac{F S}{F} \\
& \Rightarrow g \in\left\{1-\frac{F S}{F} \mid F S \in I N\right\} \vee \\
g & =g(M)=1-\frac{1}{M} \\
& \Rightarrow g \in\left\{1-\frac{1}{M} \mid M \in I N\right\}
\end{aligned}
$$

In contrast to factual correct values as written by (4), we model $g$ to take every value in the interval [0; 1 [, i.e. $0 \leq g<1$. This mathematical simplification allows a continuous objective function and thus an analytical analysis. In the following,

\footnotetext{
${ }^{1}$ Let a SU be a general measure for a size of software. Applying the model, this measure should be replaced by a software metric such as the Function Point Analysis or COCOMO.
} 
we dissolve this conflict in the operationalization by showing factual correct optimal results according to (4).

The degree of granularity has effects on the parameters of the return function. According to Assumptions 4-7, the fixed maximum demand $Q$ is replaced by the granularity dependent maximum demand $Q(g)$. The price (2) is multiplied with the price advance function $h(g)$. The total costs are the sum of production and reproduction costs and are written as $C=C_{p}+C_{g}(g)+C_{r}(x)$.

As granularity has effects on the mentioned parameters and as these parameters are multiplied with the demand $x$ in the return function, demand and degree of granularity are dependent. Thus, there is a return maximizing demand which itself depends on the granularity and is denoted by $x^{*}(g)$. In the following, this relation is always employed instead of the variable $x$. By integrating these effects, the return function (3) can be written depending on $g$ and is used as objective function:

$$
\begin{aligned}
& R\left(x^{*}(g), g\right) \\
& =\frac{Q(g)-x^{*}(g)}{\beta} \cdot h(g) \cdot x^{*}(g) \\
& \quad-C_{p}-C_{g}(g)-C_{r}\left(x^{*}(g)\right) \rightarrow \max ! \\
& 0 \leqq g<1
\end{aligned}
$$

By concretizing this general form of the objective function, we will now examine the effects of granularity.

\subsection{Basic Decision Model}

Based on the general form, we define a simplified setting that enables an analytical examination of the fundamental effects caused by granularity. We therefore assume that granularity causes linear changes on the model parameters demand, price and costs for granularity. Additionally, in this stage of the model, we still assume that reproduction costs are fixed as in CSP models. Based on the assumptions of the previous subsection and replacing them, we make new simplified assumptions:

Assumption 4.1 The maximum demand may increase, stagnate or decrease, and is expected to vary linearly on the modification factor $\eta$ with increasing degree of granularity.

$Q(g)=Q \cdot(1+\eta \cdot g)$
Assumption 5.1 The price per single SU increases linearly by the modification factor $\gamma>0$ with increasing degree of granularity.

$h(g)=1+\gamma \cdot g$

Assumption 6.1 Costs for implementing and maintaining functionality are fixed (denoted by $P C$ ). Costs for granularity increase linearly up to the maximum extent $G C$.

$C_{p}=P C$

$\wedge \quad C_{g}(g)=G C \cdot g$

Assumption 7.1 Reproduction costs consist of a fixed cost block $R C$ and neglectable variable costs.

$C_{r}\left(x^{*}(g)\right)=R C$

With these new assumptions, the objective function can now be written as:

$$
\begin{aligned}
R\left(x^{*}(g), g\right) & \\
= & \frac{Q \cdot(1+\eta \cdot g)-x^{*}(g)}{\beta} \\
& \cdot(1+\gamma \cdot g) \cdot x^{*}(g) \\
& \quad-P C-G C \cdot g-R C \rightarrow \max ! \\
& 0 \leqq \\
& \quad<1
\end{aligned}
$$

To obtain a relation $x^{*}(g)$ between optimal demand and granularity, we set the 1st partial derivative of the return function with respect to the demand to 0 . By solving the resulting equation for the demand and checking the 2nd order condition, we can write the return maximizing demand as a function of the degree of granularity (Simon and Blume 1994, cp. Appendix A):

$x^{*}(g)=\frac{Q \cdot(1+\eta \cdot g)}{2}$

Inserting this relation into (10), the optimization problem is written as:

$$
\begin{aligned}
R(g)= & \frac{Q^{2} \cdot(1+\eta \cdot g)^{2}}{4 \cdot \beta} \cdot(1+\gamma \cdot g) \\
& -P C-G C \cdot g-R C \rightarrow \max ! \\
0 \leqq & g<1
\end{aligned}
$$

\subsubsection{Model Analysis}

We will now examine the model analytically and deduce general statements concerning the model parameters. As the maximum demand may increase, stagnate or decrease due to changes of granularity, two cases have to be considered. Before stepping into the analysis, we can state that providers should abstain from offering granular services if costs for granularity are very high and exceed possible additional income. This is true for both of the following cases.

Case 1: Maximum demand increases or stagnates with increasing granularity $(\eta \geqq 0)$

Very simple and intuitive statements can be deduced for this case. With increasing granularity, prices will increase and the demand will stagnate or increase. As income (as product of prices and demand) will always increase if more granular services are offered, providers should always pick the maximum degree of granularity.

Case 2: Maximum demand decreases with increasing granularity $(\eta<0)$

With increasing granularity, prices will increase and the maximum demand will decrease. Thus, in contrast to Case 1, the maximum income and thus the degree of granularity may be situated anywhere in the feasible interval. We now seek to derive the optimal degree of granularity; a more detailed derivation is shown in Appendix B.

To obtain a possible optimal degree of granularity which we denote with $\hat{g}$, the 1st order condition for $\hat{g}$ is:

$$
\begin{aligned}
\frac{\partial R}{\partial g}= & -\frac{\eta_{d} \cdot Q^{2} \cdot\left(1-\eta_{d} \cdot g\right) \cdot(1+\gamma \cdot g)}{2 \cdot \beta} \\
& +\frac{\gamma \cdot Q^{2} \cdot\left(1-\eta_{d} \cdot g\right)^{2}}{4 \cdot \beta}-G C \\
\stackrel{!}{=} & 0 \rightarrow \hat{g}
\end{aligned}
$$

The 2nd order condition also has to be satisfied for $\hat{g}$ to determine a unique return maximum:

$$
\begin{aligned}
\left.\frac{\partial^{2} R}{\partial g^{2}}\right|_{g=\hat{g}}= & -\frac{\eta_{d} \cdot \gamma \cdot Q^{2} \cdot\left(1-\eta_{d} \cdot g\right)}{\beta} \\
& +\frac{\eta_{d}^{2} \cdot Q^{2} \cdot(1+\gamma \cdot g)}{2 \cdot \beta} \stackrel{!}{<} 0
\end{aligned}
$$

Based on the 1st derivative, we can determine two possible optima, of which this one satisfies the 2 nd order condition:

$$
\begin{aligned}
\hat{g}= & \frac{2}{3 \cdot \eta_{d}}-\frac{1}{3 \cdot \gamma} \\
& -\frac{\sqrt{\frac{4}{3} \cdot G C \cdot \beta+\frac{1}{9} \cdot Q^{2} \cdot\left(\eta_{d}^{2}+2 \cdot \eta_{d} \cdot \gamma+\gamma^{2}\right)}}{\eta_{d} \cdot \gamma \cdot Q}
\end{aligned}
$$

The parameter $\eta$ has been substituted by $\eta_{d}=-\eta$. As now all parameters in the 
following equation are positive, the analysis is easier to follow.

As the decision variable is defined in an interval, the possible optimum has to be proved for feasibility and the position of $\hat{g}$ has to be examined. If $\hat{g}$ is situated within the interval, it is the optimal $g^{*}$. If $\hat{g} \geq 1$, one should select the maximum granularity. In case of $\hat{g}<0$, the return maximizing granularity is either at the lower or upper boundary (no or maximum granularity), thus both points have to be examined (cp. Appendix B).

These derivations and formulas (especially (15)) form the foundation for analyzing the real world case. At this point, we can deduce the following general statements concerning the behavior of the degree of granularity due to variations of the parameters.

The first findings concerning the parameters that are directly conjunct with granularity are very intuitive: Lower costs for granularity (lower GC) and a higher WTP for more specific functionality ( $\gamma$ increases) may lead to an increasing degree of granularity. In contrast, the more customers only request few specific modules $\left(\eta_{d}\right.$ increases (or $\eta$ decreases)), the more the degree of granularity should decrease. Thus, by offering services of lower granularity, providers can ensure to sell a critical mass of SU by offering larger parts and thus force customers to buy more $\mathrm{SU}$ than requested. This is also true if large parts of the functionality are requested by the majority of customers, and customers requesting only small parts must not be given much attention.

Even more interesting are general statements on given market parameters: A larger $\beta$, i.e. lower general WTP and more inelastic demand coming up with lower customers' reservation prices, would lead to lower granularity. This is generally due to lower attainable prices. Providers may try to compensate this by selling larger services containing more SU. Second, a larger market/higher maximum demand (larger $Q$ ) causes not only higher sales volume and prices (Varian 2009), but also higher granularity. This is due to rising income (since more functionality can be sold and prices will increase) in contrast to constant costs for granularity. Therefore, providers should try to influence the market size in a positive manner: Starting points would be e.g. a higher focus on customer relationship management (CRM) to support customer retention and acquisition, or to expand the amount of offered functionality to sell more SU per customer. However, it must be overhauled if there is demand for such expansions and if this demand is not already covered by another provider.

\subsubsection{Operationalization: Introduction of the Real World Case}

This case will serve as a running example to further illustrate the application of the model and is based on data of the SaaS provider IESP. Names as well as all identifying details are omitted and the business case data have been anonymized and slightly abridged for reasons of confidentiality.

Besides SaaS suites for CRM and financials, IESP offers an independent suite for employee resource management (ERM). Furthermore, a technical module runs in the background which is required for all three suites. The real world case is based on data of this ERM functionality. Due to its very high functional maturity the ERM software nearly has an exclusive position on the respective SaaS market. The suite contains the modules self services for employees/managers, human resources management and document management.

IESP employs the software metric Function Point Analysis to measure the size as this method allows a detailed analysis (Albrecht 1979; Jones 2007). The functionality has been estimated to 20,000 function points with this method based on content and complexity of included functions. Based on historical data, implementation costs for function updates and maintenance are estimated to $€ 2.6$ million. Costs for provision including user support are estimated at $€ 400,000$.

IESP has recently offered the ERM suite in a single service, but is contemplating to change its strategy: By offering the functionality within more granular services, new customers shall be acquired who demand only certain parts of the whole ERM suite. The potential of such a strategic change has been evaluated with a market study which is based on internal data and purchased data from a market research institute.

IESP has internal data about recent customers and potential customers who have been subject to acquisition in the last two years. These data comprise the number of requested user licenses, requested parts of the suite and WTP.

The data from the institute were based on a questionnaire in which developments of SaaS markets and its segments (e.g. CRM, ERM) were estimated and which was sent to a wide range of large and medium-sized companies. For each segment, the questionnaire contained a list of functionalities that the requested software should provide. Participating firms were asked to mark the functionality they require and to estimate the number of licenses. Furthermore, they should quantify the extent of WTP if they bought software satisfying their requirements to a large extent (e.g. standard software) or fully (e.g. customized software). Concerning the ERM functionality, the external data give information about customers, potential sales volume of ERM subfunctions and attainable prices.

From the external data, IESP selected data concerning its target groups. These data were matched with internal data. Concerning demand, potential customers are segmented into five clusters (cp. Table 2). Clusters C1-C3 consist of

Table 2 Annual market potential

\begin{tabular}{|c|c|c|c|c|c|c|}
\hline \multirow[t]{2}{*}{ Attribute } & \multirow[t]{2}{*}{ Symbol/Calculation } & \multicolumn{5}{|c|}{ Cluster c } \\
\hline & & $\mathrm{C} 1$ & $\mathrm{C} 2$ & $\mathrm{C} 3$ & $\mathrm{C} 4$ & $\mathrm{C} 5$ \\
\hline Number of customers [users/year] & $N_{c}$ & 1,000 & 1,500 & 2,500 & 3,000 & 4,000 \\
\hline Required functionality [\%] & $R E Q_{c}$ & $100 \%$ & $65 \%$ & $50 \%$ & $25 \%$ & $15 \%$ \\
\hline Required functionality [SU] & $R E Q_{c} \cdot F$ & 20,000 & 13,000 & 10,000 & 5,000 & 3,000 \\
\hline Maximum WTP per customer $[€]$ & $W T P_{c}$ & 3,000 & 2,200 & 1,750 & 900 & 570 \\
\hline Maximum WTP per SU and customer [€/SU] & $\frac{W T P_{c}}{F \cdot R E Q_{c}}$ & 0.15 & 0.169 & 0.175 & 0.18 & 0.19 \\
\hline
\end{tabular}


Table 3 Parameters of the model

\begin{tabular}{|c|c|c|}
\hline Parameter & Formula & Value \\
\hline$Q(0)[S U]$ & $\sum_{c=1}^{3} N_{c} \cdot F$ & $(1,000+1,500+2,500) \cdot 20,000=100,000,000$ \\
\hline$Q(0 . \overline{9})[\mathrm{SU}]$ & $\sum_{c=1}^{5} N_{c} \cdot R E Q_{c} \cdot F$ & $\begin{array}{l}(1,000 \cdot 1.0+1,500 \cdot 0.65+2,500 \cdot 0.5+3,000 \cdot 0.25+4,000 \cdot 0.15) \cdot 20,000 \\
\quad=91,500,000\end{array}$ \\
\hline $\begin{array}{l}\text { Maximum } P R \text { per } S U \\
\text { and customer for } g=0 \\
{[€ / S U]}\end{array}$ & $\max \left(\frac{W T P_{c}}{F}\right)$ & $\frac{3,000}{20,000}=0.15$ \\
\hline $\begin{array}{l}\text { Maximum } P R \text { per } S U \\
\text { and customer for } g=0 . \overline{9} \\
{[€ / S U]}\end{array}$ & $\max \left(\frac{W T P_{c}}{F \cdot R E Q_{c}}\right)$ & $\frac{570}{20,000 \cdot 0.15}=0.19$ \\
\hline$\beta$ & $\frac{Q(0)}{P R(0)}$ & $\frac{100,000,000}{0.15}=666,666,666$ \\
\hline$P C[€]$ & - & $2,600,000$ \\
\hline$R C[€]$ & - & 400,000 \\
\hline$G C[€]$ & - & 175,000 \\
\hline$\eta$ & $1-\frac{Q(0 . \overline{9})}{Q(0)}$ & $1-\frac{91,500,000}{100,000,000}=-0.085$ \\
\hline$\gamma$ & $\frac{P R(0 . \overline{9})}{P R(0)}-1$ & $\frac{0.19}{0.15}-1=0.2667$ \\
\hline
\end{tabular}

potential customers for the already offered full version, C4 and C5 of new potential customers which are interested in parts of the suite and only could be addressed with services of higher granularity. For estimating the demand of $\mathrm{C} 1-\mathrm{C} 3$, primarily internal data were taken. As the external data show higher sales potential, estimations were made to a certain extent, but for reasons of safety not reaching the full extent as predicted by the external data. For C4 and C5 external data were taken and modified by a safety reduction. Concerning WTP, lower and upper ranges result from data for each cluster. As the reservation price (RP) is derived from the maximal WTP, and resulting market prices in monopolies are usually lower than RP, for reasons of clearness only the upper range of the WTP is listed in Table 2.

IESP now has evidence concerning the potential number of users and their specific amount of required functionality as well as to their maximum WTP for using the functionality. We have also labeled every attribute with a symbol, as these figures are used to calculate the model parameters and the calculation is easier to follow. Costs for granularity, i.e. for spreading functionality over more services, are estimated to rise to a maximum sum of $€ 175,000$.

Together with experts of IESP, we took these figures to quantify the model pa- rameters. First, individual demands and WTP had to be aggregated to the demand curve. For a monopoly (Varian 2009), the RP equals the maximum individual WTP, and maximum demand is an aggregation of all individual demands, i.e. potential salable SU of relevant clusters are added. Second, we assumed that varying the granularity influences the market, and hence demand curve and its parameters. As we assumed linear progression due to granularity, we had to quantify the maximum demand, reservation prices and costs for minimum (all recent customer clusters $\mathrm{C} 1-\mathrm{C} 3$ are relevant) and maximum (all clusters are relevant) granularity. Based on demand and prices, we could determine modification factors and market parameters ${ }^{2}$ as listed in Table 3.

Using the model based on these parameters, it suggests an optimal granularity and a market price as well as the amount of sold SU. ${ }^{3}$

\subsubsection{Optimization}

Inserting the parameters of Table 3 into (15), the optimal degree of granularity can be calculated analytically. Figure 1 and Table 4 show the optimal results with relevant economic data. To show the effects of granularity, we also list the values for no granularity.
First, this result has to be proved for feasibility. Examining the result of $\hat{g}=0.69$ (cp. Model Analysis; Appendix B) reveals that this $\hat{g}$ is also the optimal theoretical $g^{*}$ representing 3.2 services. The next $g$ with an integer number of services (cp. (4)), which also has the highest return, is $g_{\text {int }}^{*}=0.667 \mathrm{im}$ plying the functionality should be split on three services, and now called integer maximum. This new allocation causes significant changes in the amount of sold functionality decreasing by $6 \%$ and prices attained per SU increasing by $11 \%$. These effects are due to a change in the customer structure. Though, the customer base can be enlarged, the sales volume decreases as services are smaller and more specific and customers only buy required functionality. In the context of this example, the proposed degree of granularity would imply in particular customers of clusters C2 and C3 and limited C4 could be provided with services more specific to their demand. Furthermore, customers' WTP can be better skimmed resulting in higher prices per SU since the offered smaller services are more specific to their demand. This results in higher income. Compared to the origin state, where only a single service was offered, the income increases by $€ 180,367$. The costs only increase by $€ 116,666$. In sum, return would increase by $€ 63,701$ or $8 \%$, respectively.

\footnotetext{
${ }^{2}$ Due to changing granularity, other customer clusters with different required SU and WTP may become relevant for determining $\beta$ resulting in another value for $\beta$. However, $\beta$ is kept fixed and these effects were modeled by the factor $\gamma$ which itself is multiplied with $\beta$ in the objective function.

${ }^{3}$ Due to characteristics of a monopoly, both market price and sold SU will be lower than maximal RP and demand. Thus, resulting prices and demand may differ from the estimations.
} 
Fig. 1 Graphical representation of the results

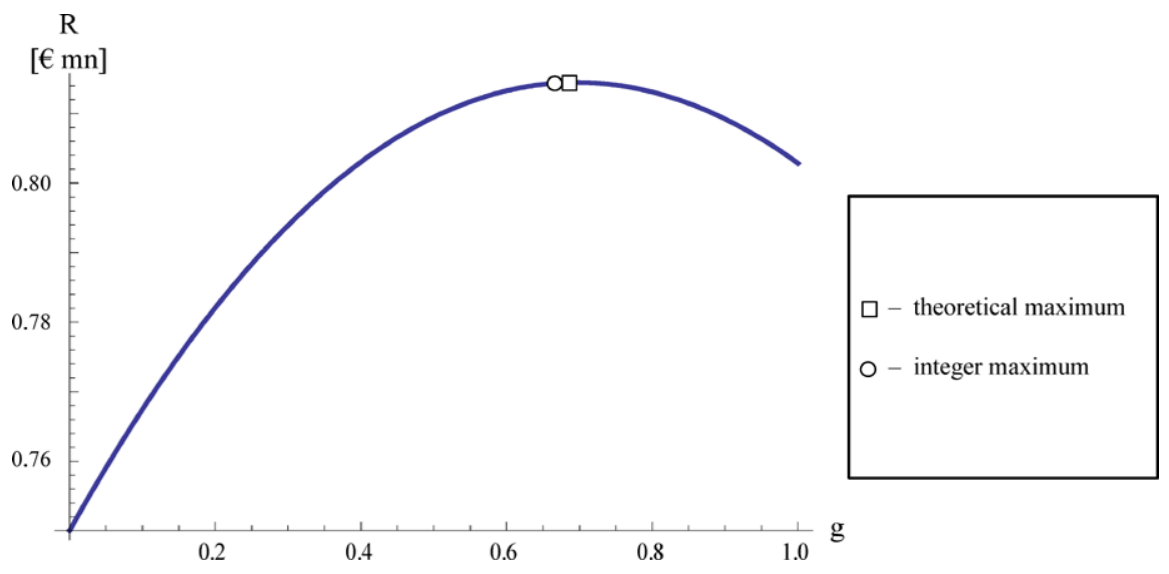

Table 4 Optimization results

\begin{tabular}{|c|c|c|c|c|c|c|c|}
\hline$g$ & Return $[€]$ & Income $[€]$ & Costs $[€]$ & $\begin{array}{l}\text { Number } \\
\text { of Services }\end{array}$ & $\begin{array}{l}\text { Offer share } \\
{[\%]}\end{array}$ & Sold SU & $\begin{array}{l}\text { Price per } \\
\text { SU }[€]\end{array}$ \\
\hline$g^{*}=0.69$ & 813,788 & $3,935,021$ & $3,121,233$ & 3.2 & 31 & $47,055,777$ & 0.084 \\
\hline$g_{\text {int }}^{*}=0.667$ & 813,701 & $3,930,367$ & $3,116,666$ & 3 & 34 & $47,166,666$ & 0.083 \\
\hline$g=0$ (no granularity $)$ & 750,000 & $3,750,000$ & $3,000,000$ & 1 & 100 & $50,000,000$ & 0.075 \\
\hline
\end{tabular}

We can state in the context of this example that a provider can enlarge its return by offering granular services, though the amount of sold functionality may decrease. This positive economic effect is due to a win-win setting for customers and provider enabled by granularity: Customers can purchase functionality highly specific to their demand and spend the amount they are willing to pay for required functionality. Providers can realize higher prices per module and enlarge the business value of their functionality. This effect is even increased by the characteristics and payment modalities of SaaS, since customers pay per use or per period for a bundle of infrastructure and functionality and do not have to install software on own servers. Therefore, customers are very flexible and variable in costs since they only have low fixed costs for initial investments (Sääksjärvi et al. 2005, p. 183). As the impact of upfront costs decreases, fine-grained SaaS functionality is in particular attractive for customers with little or medium demand. Thus, providers should use granularity as an instrument to attract new customers and attain higher income.

\subsection{Model Extension - Reproduction Costs}

In this stage, let us examine effects of reproduction costs as postulated (cp. Modeling Issue 5). Until now, we have as- sumed fixed reproduction costs. As communication and calculation costs increase with the amount of sold functionality (Lehmann and Buxmann 2009), we replace Assumption 7.1 by:

Assumption 7.2 Reproduction costs increase linearly with the amount of sold functionality.

Thus, the more functionality is sold and the more following customers are acquired, the higher reproduction costs will be. They can be calculated as product of costs per single sold SU $R C_{\mathrm{SU}}$ and actual demand.

$C_{r}\left(x^{*}(g)\right)=R C_{\mathrm{SU}} \cdot x^{*}(g)$

With this additional assumption, the objective function is now written as follows:

$$
\begin{aligned}
R\left(x^{*}(g), g\right)= & \frac{Q \cdot(1+\eta \cdot g)-x^{*}(g)}{\beta} \\
& \cdot(1+\gamma \cdot g) \cdot x^{*}(g)-P C \\
& -G C \cdot g-R C_{\mathrm{SU}} \\
& \cdot x^{*}(g) \rightarrow \max ! \\
0 \leqq g<1 &
\end{aligned}
$$

This enhancement complicates the return function, and now it is no longer possible to solve the maximization problem analytically. We have to solve the problem numerically. We implemented and solved (17) with the 'NMaximize' function of the program 'Mathematica'.
Operationalization: Continuation of the Real World Case In the previous stage, we underlaid fixed reproduction costs of $€ 400,000$. Together with experts of IESP, we analyzed the relevant costs and estimate the maximum reproduction costs, in case the demand would be fully covered, at $€ 600,000$ (best case) or $€ 800,000$ (worst case), respectively. This result in a parameter value for $R C_{\mathrm{SU}}$ of 0.006 or 0.008 [both in $€ / S U$ ]. To exemplify the effects of reproduction costs, we calculate both cases. Table 5 lists the results of the optimization.

Figure 2 shows the curve progressions and maxima for both scenarios.

As both $g^{*}$ are feasible and granularity is also attractive as in the previous section, even with increases in return reaching $13 \%$, we direct our analysis towards one main question: Which differences occur if reproduction costs are considered (which is usually neglected in models for CSP)? We can see that reproduction costs foster higher granularity and moreover return can be increased. This is also depicted in Fig. 2 where the maximum of the return curve moves toward higher granularity for both cases. This has two reasons: reproduction costs decrease with higher granularity, and providers can simultaneously exploit the value of their services due to higher attainable prices. This is economically sensible as long as the reduction of reproduction costs and the increase in income exceed costs for 
Table 5 Optimization results

\begin{tabular}{|c|c|c|c|c|c|c|c|c|c|}
\hline $\begin{array}{l}R C_{\mathrm{SU}} \\
{[€ / \mathrm{SU}]}\end{array}$ & $g$ & $\begin{array}{l}\text { Return } \\
{[€]}\end{array}$ & $\begin{array}{l}\text { Income } \\
{[€]}\end{array}$ & $\begin{array}{l}\text { Total costs } \\
{[€]}\end{array}$ & $\begin{array}{l}\text { Reproduction } \\
\text { costs }[€]\end{array}$ & $\begin{array}{l}\text { Number of } \\
\text { Services }\end{array}$ & $\begin{array}{l}\text { Offer share } \\
{[\%]}\end{array}$ & Sold SU & $\begin{array}{l}\text { Price per } \\
\text { SU }[€]\end{array}$ \\
\hline \multirow[t]{3}{*}{0.006} & $g^{*}=0.79$ & 938,495 & $3,947,036$ & $3,008,541$ & 269,892 & 4.8 & 21 & $44,982,002$ & 0.088 \\
\hline & $g_{\text {int }}^{*}=0.8$ & 938,452 & $3,948,164$ & $3,009,712$ & 269,712 & 5 & 20 & $44,977,000$ & 0.088 \\
\hline & $g=0$ & 850,000 & $3,750,000$ & $2,900,000$ & 300,000 & 1 & 100 & $50,000,000$ & 0.075 \\
\hline \multirow[t]{3}{*}{0.008} & $g^{*}=0.82$ & 849,162 & $3,947,692$ & $3,098,530$ & 354,532 & 5.6 & 18 & $44,316,571$ & 0.089 \\
\hline & $g_{\mathrm{int}}^{*}=0.833$ & 849,149 & $3,949,198$ & $3,100,049$ & 354,216 & 6 & 17 & $44,278,300$ & 0.089 \\
\hline & $g=0$ & 750,000 & $3,750,000$ & $3,000,000$ & 400,000 & 1 & 100 & $50,000,000$ & 0.075 \\
\hline
\end{tabular}

Fig. 2 Graphical representation of the results

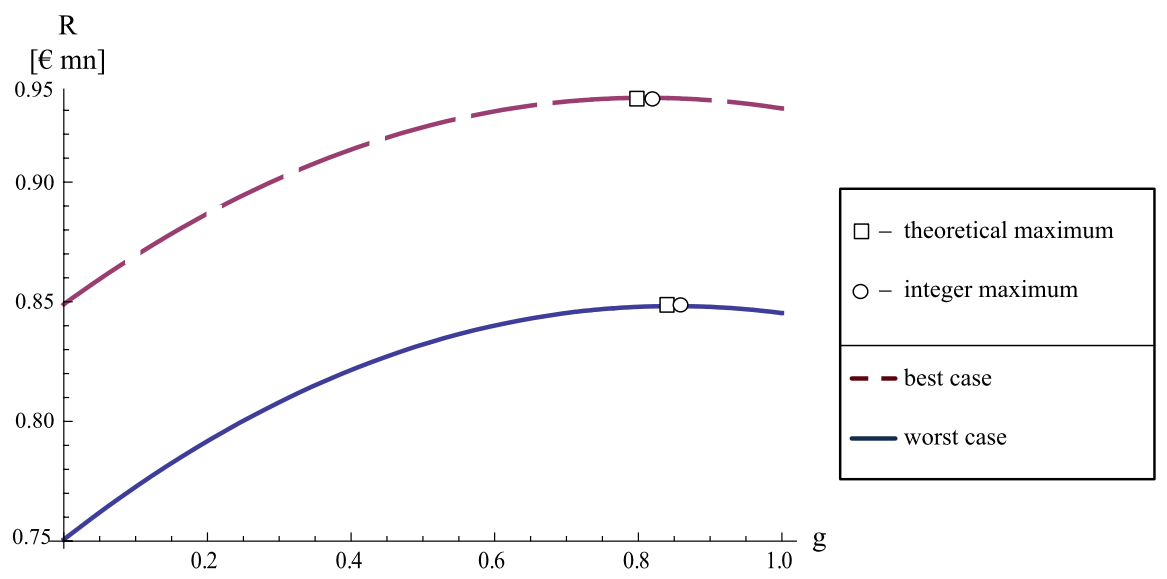

granularity. Thus, providers should carefully weigh the characteristics of these factors against each other. By comparing both cases, one can say that higher reproduction costs result in higher granularity. If a provider has functionality with high data traffic or intensive computations, it might be interesting to reduce reproduction costs by further specializing the offer with more granular services. Another aspect is the development of hardware prices which are steadily decreasing and thus reduce reproduction costs (cp. best case). Here we can state that lower reproduction costs have decreasing effects on the degree of granularity. In conclusion, we have to point out that considering variable reproduction costs for SSP models is of crucial importance. As implication for scholars, let us emphasize that reproduction costs cannot be neglected in models for SaaS as done in models for CSP.

\section{Discussion of Limitations and Practical Application}

The introduced model is based on a set of assumptions. We explain why the model delivers valid results though built on rigid assumptions and discuss how their relaxation can improve practical impact. Furthermore, these limitations at the same time bear extension potential.

We assumed a monopoly as this market form is often employed in theory since it allows for a good analysis of effects on duopoly or competition are also conceivable as providers may offer similar software and thus have to compete for market shares. Concerning these other market forms, it seems that firms exercising certain market power, e.g. price leaders, may profit from similar effects like a monopolist. In this case, findings may be partially transferred. In contrast, if firms have no influence on the market, transferability of findings is hindered. Thus, other market forms could be subject to further research.

We introduced a rigid assumption that the functionality has to be cut into services of same size. This implies that regarded functionality has to consist of several functional blocks that can be offered independently of each other, whereby cuts have to be made along these blocks. Though in reality modules are usually not of same size, the model may offer 125 a guide value which granularity should be the market. Other market forms such as a chosen for software of concurrent modules. Table 6 illustrates how the degree of granularity can be mapped to cutting functionality.

A related problem is finding a sufficient size that still provides enough value for customers. For instance, it might be not reasonable to offer functionality which only returns a list of employees. Instead, a minimum size would be to combine this listing function at least with timesheet tracking or expense reports. In sum, though decision makers have to define a minimum size, the model can support them in finding a size equal to or above this defined lower limit.

Increasing granularity is followed by increasing prices. However, customers requesting all functionality would have to pay more with higher granularity, in spite of purchasing the same amount of functionality. This bears the risk of the loss of such customers. Including volume discounts for customers requesting all or most functionality may be a starting point for this extension.

The model captures basic effects in an aggregate approach which enabled showing fundamental aspects of the problem in a comprehensible way. However, income and costs may differ from service to service as e.g. some services cause a larger 
Table 6 Interpretation of granularity

\begin{tabular}{llll}
\hline Granularity & Description & Number of Services & Interval for $g$ \\
\hline $\begin{array}{l}\text { No granularity } \\
\text { Low granularity }\end{array}$ & $\begin{array}{l}\text { One service comprising all SU. } \\
\text { The functionality is split into its major parts: } \\
\text { employee self services, HR management and } \\
\text { document management. }\end{array}$ & $2-3$ & [0.0; 0.5[ \\
Medium granularity & $\begin{array}{l}\text { Major parts are split into their main } \\
\text { functionalities. For instance, HR management } \\
\text { could be split into employee administration, } \\
\text { acquisition, reporting, and payroll. } \\
\text { The functionality is split into services of an } \\
\text { economically reasonable minimal size. }\end{array}$ & $40.667\left[\begin{array}{l}0.667 ; 0.917[ \\
\text { High granularity }\end{array}\right.$ & $>12$ \\
\hline
\end{tabular}

data transfer than others (Boerner and Goeken 2009) or some parts of the functionality are in higher demand. In practice, a more detailed analysis with a disaggregation to single services may be appropriate. These parameter estimations based on single services can be aggregated and then employed to our model. Further research could deal with other analysis methods such as two-(or more-)part pricing schemes to consider heterogeneity of services.

Finally, we assumed linear changes due to varying granularity. While this allowed a meaningful analysis by an analytical solution, the applicability of linear changes is limited. This is especially true for granularity costs, as the number of interfaces may crucially increase (Heinrich and Fridgen 2005). Such an enormous increase would result in exponentially rising costs. In this case, our model would propose a lower degree of granularity compared to linear progression. However, the general form can be easily substantiated with arbitrary realistic, e.g. exponential relations, or with more detailed input data as mentioned in the previous paragraph.

\section{Conclusion}

SaaS bears much economic potential, but there are only few quantitative decision support models for this uprising provisioning type. In particular, there is a lack of research concerning product differentiation of SaaS. To contribute to filling this gap, we presented a formal approach considering aspects of product differentiation for SaaS; in particular effects on prices, sales volume and costs were considered. We discussed applicability of existing CSP versioning models to SaaS and identified modeling issues, in particular that service granularity variation is a viable instrument of product differentiation for SaaS. Starting out with micro-economic models based on the demand curve, we elaborated which effect varying granularity has on the market and hence on parameters of the demand curve. Then we included these effects into the parameters of the demand curve and presented a decision model based on this extended demand curve. Furthermore, we included variable reproduction costs which have mostly been neglected in quantitative research so far. Finally, we illustrated our approach with a real world case.

The article formalized concepts of product differentiation for SaaS and should help to establish basic understanding in this area. Thus, our approach provided insights into the economic trade-off between the major influence factors. In analyzing the presented model, relations between these major influence factors and varying a SaaS product could be deducted. Furthermore, the model provides evidence that reproduction costs may have significant influence on granularity and profits. Thus, they should necessarily be considered for SSP models. This is contrary to prior versioning approaches for CSP. In summary, the proposed model for supporting decisions concerning service offerings not only formalized this highly relevant decision problem, it can also form the foundation for further research.

\section{Appendix A: Relation Between the Degree of Granularity and the Actual Demand}

This appendix contains a detailed derivation of the relation between the degree of granularity and the actual demand.
The actual demand $x$ and the degree of granularity g are mathematically dependent as they are multiplied in the objective function:

$$
\begin{aligned}
R\left(x^{*}(g), g\right)= & \frac{Q \cdot(1+\eta \cdot g)-x^{*}(g)}{\beta} \\
& \cdot(1+\gamma \cdot g) \cdot x^{*}(g)-P C \\
& -G C \cdot g-R C \rightarrow \max !
\end{aligned}
$$$$
0 \leqq g<1
$$

To allow an analysis and resulting general statements based on an analytical solution, we have to determine a relation $x^{*}(g)$ between optimal demand and granularity to obtain an objective function depending one a single variable. Therefore, the 1st partial derivative of the return function $((10)$ in the paper, or here: (A.1)) with respect to the demand has to be set to 0 :

$$
\begin{aligned}
\frac{\partial R}{\partial x^{*}(g)}= & {[(Q \cdot(1+\eta \cdot g)} \\
& \left.\left.-2 \cdot x^{*}(g)\right) \cdot(1+\gamma \cdot g)\right] \beta^{-1} \\
\stackrel{!}{=} & 0 \rightarrow x^{*}(g)
\end{aligned}
$$

By solving the resulting equation for the regarded case, we receive the return maximizing relation between actual demand and degree of granularity (cp. (11) or (A.3)):

$x^{*}(g)=\frac{Q \cdot(1+\eta \cdot g)}{2}$

Finally, this resulting relation must be checked with the 2 nd order condition:

$$
\frac{\partial^{2} R}{\partial\left(x^{*}(g)\right)^{2}}=-\frac{2+2 \cdot \gamma \cdot g}{\beta} \stackrel{!}{<} 0
$$

The 2nd order condition is true for the calculated relation. Hence, it can be used as maximizing relation between actual demand and degree of granularity and can be further employed in the objective function. 


\section{Abstract}

\section{Arne Katzmarzik}

\section{Product Differentiation for Software-as-a-Service Providers}

The market for the new provisioning type Software-as-a-Service (SaaS) has reached a significant size and still shows enormous growth rates. By varying size of SaaS products, providers can improve their market position and profits by successfully acting in the tension area of customer acquisition, pricing and costs. We first elaborate differences concerning product differentiation between classic software provisioning models and SaaS. Then, we introduce a micro-economic based decision model to maximize the return of a provider by finding an optimal granularity, i.e. by varying the size of services. This paper makes two contributions in this context: (1) it provides a conceptual foundation for product differentiation within the scope of SaaS and (2) it presents the first implementation of variable reproduction costs for web based software offers. The model is illustrated by a real world case with data from a SaaS provider.

Keywords: Software-as-a-Service, Product differentiation, Service granularity, Decision model

\section{Appendix B: Detailed Derivation of the Optimal Degree of Granularity}

This appendix contains a detailed derivation of the optimal degree of granularity in case of decreasing maximum demand with increasing granularity (cp. 3.2 - Case 2).

Starting out with the objective function (12), the possible optimal degree of granularity, which we denote with $\hat{g}$, can be calculated over the 1st order condition.

$$
\begin{aligned}
\frac{\partial R}{\partial g}= & -\frac{\eta_{d} \cdot Q^{2} \cdot\left(1-\eta_{d} \cdot g\right) \cdot(1+\gamma \cdot g)}{2 \cdot \beta} \\
& +\frac{\gamma \cdot Q^{2} \cdot\left(1-\eta_{d} \cdot g\right)^{2}}{4 \cdot \beta}-G C \\
\stackrel{!}{=} & 0 \rightarrow \hat{g} \quad \text { (B. } 1)
\end{aligned}
$$

Based on the 1st derivative, two possible optima can be determined. For reasons of simplicity we also substitute the parameter $\eta$ by $\eta_{d}=-\eta$ as now all parameters in the following equation are positive and the derivation is easier to understand.

$$
\begin{aligned}
\hat{g}_{1}= & \frac{2}{3 \cdot \eta_{d}}-\frac{1}{3 \cdot \gamma} \\
& -\frac{\sqrt{\frac{4}{3} \cdot G C \cdot \beta+\frac{1}{9} \cdot Q^{2} \cdot\left(\eta_{d}^{2}+2 \cdot \eta_{d} \cdot \gamma+\gamma^{2}\right)}}{\eta_{d} \cdot \gamma \cdot Q} \wedge \\
\hat{g}_{2}= & \frac{2}{3 \cdot \eta_{d}}-\frac{1}{3 \cdot \gamma} \\
& +\frac{\sqrt{\frac{4}{3} \cdot G C \cdot \beta+\frac{1}{9} \cdot Q^{2} \cdot\left(\eta_{d}^{2}+2 \cdot \eta_{d} \cdot \gamma+\gamma^{2}\right)}}{\eta_{d} \cdot \gamma \cdot Q}
\end{aligned}
$$

The next step is to analyze the calculated extrema. As the objective function for this case is a polynomial of 3rd degree, there is always one local maximum and one local minimum (Simon and Blume 1994). Thus, for determining a unique return maximum the 2 nd order condition must be satisfied for the possible optima $\hat{g}$ :

$$
\begin{aligned}
\left.\frac{\partial^{2} R}{\partial g^{2}}\right|_{g=\hat{g}} & \\
= & -\frac{\eta_{d} \cdot \gamma \cdot Q^{2} \cdot\left(1-\eta_{d} \cdot g\right)}{\beta} \\
& +\frac{\eta_{d}^{2} \cdot Q^{2} \cdot(1+\gamma \cdot g)}{2 \cdot \beta} \stackrel{!}{<0}
\end{aligned}
$$

As all parameters are positive, $\hat{g}_{2}$ is always greater than $\hat{g}_{1}$. By inserting both possible optima into the 2 nd derivative, we can state that the value of the 2 nd derivative at $\hat{g}_{2}$ is always greater than the value at $\hat{g}_{1}$. According to the 2 nd order condition, the local maximum must be situated at the lower value, i.e. $\hat{g}_{1}$, and the local minimum at the greater value, i.e. $\hat{g}_{2}$. Thus, the possible return maximum which has to be examined for feasibility is: $\hat{g}=\hat{g}_{1}$.

As the decision variable is defined in an interval, the position of the possible maximum has to be proven for feasibility, i.e. laying in $[0 ; 1$ [ or not. This examination can be made from the perspective of the number of services $(0<M \leq F)$

for $\hat{g} \in\left[0 ; 1\left[: \quad g^{*}=\hat{g}\right.\right.$

for $\hat{g} \geqq 1: \quad g^{*}=\lim _{M \rightarrow F^{-}} g(M)$

for $\hat{g}<0$ :

$g^{*}=0$

$$
\text { if } R(0) \geqq R\left(\lim _{M \rightarrow 1^{+}} g(M)\right)
$$

$g^{*}=\lim _{M \rightarrow F^{-}} g(M)$,

$$
\text { if } R(0)<R\left(\lim _{M \rightarrow 1^{+}} g(M)\right)
$$

or the size per service $(F \geq F S>0)$

for $\hat{g} \in\left[0 ; 1\left[: \quad g^{*}=\hat{g}\right.\right.$

for $\hat{g} \geqq 1: \quad g^{*}=\lim _{F S \rightarrow 1^{+}} g(F S)$

for $\hat{g}<0$ :

$g^{*}=0$,

$$
\begin{gathered}
\text { if } R(0) \geqq R\left(\lim _{F S \rightarrow 1^{+}} g(F S)\right) \\
g^{*}=\lim _{F S \rightarrow 1^{+}} g(F S), \\
\text { if } R(0)<R\left(\lim _{F S \rightarrow 1^{+}} g(F S)\right)
\end{gathered}
$$

Now different cases may occur and have to be examined:

If $\hat{g}$ is situated within the feasible interval, the calculated value will be the return maximum, i.e. $g^{*}=\hat{g}$.

If $\hat{g} \geqq 1$, i.e. is situated even beyond the maximum feasible granularity, the return maximum will be at $g^{*}=$ $\lim _{F S \rightarrow 1^{+}} g(F S)=\lim _{M \rightarrow F^{-}} g(M)$. Thus, the maximum granularity should be chosen, i.e. services should be of a minimal size or a maximum possible number of services should be provided, respectively.

The case $\hat{g}<0$ is more complex as the local maximum occurs at a negative value. Thus, the local minimum $\hat{g}_{2}$ may be situated within the feasible interval $[0 ; 1[$. Hence, the attainable maximum return occurs either at the lower ( $g^{*}=0$, one service comprising the total functionality) or upper boundary $g^{*}=\lim _{F S \rightarrow 1^{+}} g(F S)=\lim _{M \rightarrow F^{-}} g(M)$, services have an economically reasonable minimum size, maximum possible number of services). Therefore, both points have to be examined and the one with higher return should be selected. 


\section{References}

Albani A, Keiblinger A, Turowski K, Winnewisser C (2003) Domain based identification and modelling of business component applications. In: Proc ADBIS, pp 30-45

Albrecht AJ (1979) Measuring application development productivity. In: Proc IBM applications development symposium

Anderson C (2006) The long tail. Hyperion, New York

Arsanjani A, Ghosh S, Allam A, Abdollah T, Ganapathy S, Holley K (2008) SOMA: a method for developing service-oriented solutions. IBM Systems Journal 47(3):377396

Banker RD, Datar SM, Kemerer CF (1991) A model to evaluate variables impacting the productivity of software maintenance projects. Man Sci 37(1):1-18

Benlian A (2009) A transaction cost theoretical analysis of software-as-a-service (SAAS)-based sourcing in SMBS and enterprise. In: Proc 17th European conference on information systems, Verona

Benlian A, Hess T, Buxmann P (2009) Drivers of SaaS-adoption - an empirical study of different application types. Bus Inf Syst Eng 1(5):357-369

Bhargava HK, Choudhary V (2001) Information goods and vertical differentiation. J Manage Inf Syst 18(2):89-106

Bhargava HK, Sun D (2008) Pricing under quality of service uncertainty: market segmentation via statistical QoS guarantees Eur J Oper Res 191(3):1189-1204

Boerner R, Goeken M (2009) Identification of business services - literature review and lessons learned. In: Proc 15th Americas conference on information systems, San Francisco

Chellappa RK, Shivendu S (2005) Managing piracy: pricing and sampling strategies for digital experience goods in vertically segmented markets. Information Systems Research 16(4):400-417

Cheng HK, Tang QC (2010) Free trial or no free trial: optimal software product design with network effects. Eur J Oper Res 205(2):437447

Cheng HK, Tang QC, Zhao JL (2006) Web services and service-oriented application provisioning: an analytical study of application service strategies. IEEE Trans Eng Manage 53(4):520-533

Choudhary V, Ghose A, Mukhopadhyay T, Rajan U (2005) Personalized pricing and quality differentiation. Man Sci 51(7):11201130

Cremer H, Thisse JF (1991) Location models of horizontal differentiation: a special case of vertical differentiation models. The Journal of Industrial Economics 39(4):383-390

Erl T (2005) Service-oriented architecture: concepts, technology, and design. Prentice, Upper Saddle River

Fan M, Kumar S, Whinston AB (2009) Shortterm and long-term competition between providers of shrink-wrap software and software as a service. Eur J Oper Res 196(2):661-671

Gartner (2009) Market trends: software as a service worldwide 2009-2013

Ghose A, Sundararajan A (2005) Software versioning and quality degradation? An exploratory study of the evidence. Working paper, New York University

Haesen R, Snoeck M, Lemahieu W, Poelmans $S$ (2008) On the definition of service granularity and Its Architectural Impact. In: Proc 20th international conference on advanced information systems engineering, Montpellier, pp 375-389

Heinrich B, Fridgen M (2005) Enterprise application integration. BARev 65(1):43-61

Holschke O, Rake J, Levina O (2009) Granularity as a cognitive factor in the effectiveness of business process model reuse. In Proc 7th international conference on business process management, Ulm

Jing B (2000) Versioning information goods with network externalities. In: Proc 21 st international conference on information systems, Brisbane

Jones C (2007) Estimating software costs. McGraw-Hill, New York

Jones R, Mendelson H (2005) Information goods: development, quality and competition. Discussion paper, Stanford University

Kaplan JM (2007) SaaS: friend or foe? Business Communications Review 37(6):48-53

Kim SD, Chang SH (2004) A systematic method to identify software components. In: Proc 11th Asia-Pacific software engineering conference, Busan

Krafzig D, Banke K, Slama D (2005) Enterprise SOA - service-oriented architecture best practices. Prentice, Upper Saddle River

Lee KB, Yu S, Kim SJ (2006) Analysis of pricing strategies for e-business companies providing information goods and services. Comput Ind Eng 51(1):72-78

Lehmann S, Buxmann P (2009) Pricing strategies of software vendors. Bus Inf Syst Eng 1(6):452-462

Mietzner R, Leymann F (2008) Towards provisioning the cloud: on the usage of multigranularity flows and services to realize a unified provisioning infrastructure for Saas applications. In: Proc 2008 IEEE congress on services, Hawaii

Nault BR (1997) Quality differentiation and adoption costs: the case for interorganizational information system pricing. Ann Oper Res 71:115-142

Papazoglou MP, van den Heuvel W (2007) Service oriented architectures: approaches, technologies and research issues. The VLDB Journal 16(3):389-415

Papazoglou MP, Traverso P, Dustdar S, Leymann F (2007) Service-oriented computing: state of the art and research challenges. IEEE Computer 40(11):38-45

Parnas DL (1972) On the criteria to be used in decomposing systems into modules. ComACM 15(12):1053-1058
Phillips DE (2009) The software license unveiled: how legislation by license controls software access. Oxford University Press, Oxford

Raghunathan S (2000) Software editions: an application of segmentation theory to the packaged software market. J Manage Inf Syst 17(1):87-113

Sääksjärvi M, Lassila A, Nordström H (2005) Evaluating the software as a service business model: from CPU time-sharing to online innovation sharing. In: Proc IADIS international conference e-society, Malta, pp 177-186

Shapiro C, Varian HR (1998) Versioning: the smart way to sell information. Harv Bus Rev 76(6):106-114

Simon CP, Blume L (1994) Mathematics for economists. Norton, New York

Susarla A, Barua A, Whinston AB (2009) A transaction cost perspective of the "software as a service" business model. J Manage Inf Syst 26(2):205-240

Szyperski CA (1998) Emerging component software technologies: a strategic comparison. Software - Concepts and Tools 19(1):2-10

Valente P, Mitra G (2007) The evolution of web-based optimisation: from ASP to eservices. Decis Support Syst 43(4):10961116

Varian HR (1997) Versioning information goods. Working paper, University of California, Berkeley

Varian HR (2000) Buying sharing and renting information goods. The Journal of Industrial Economics 48(4):473-488

Varian HR (2009) Intermediate microeconomics. Norton, New York

Walsh KR (2003) Analyzing the application ASP concept: technologies, economies, and strategies. Commun ACM 46(8):103107

Wang ZJ, Xu X, Zhan D (2005) A survey of business component identification methods and related techniques. International Journal of Information Technology 2(4):229-238

Weber TA (2001) Mixed differentiation of information goods under incomplete information. In: Proc 22nd international conference on information systems, New Orleans

Weber TA (2008) Delayed multiattribute product differentiation. Decis Support Syst 44(2):447-468

Wei X, Nault BR (2006) Vertically differentiated information goods: entry deterrence, rivalry clear-out or coexistence. In: Proc 2006 INFORMS conference on information systems and technology, Pittsburgh

Zhang J, Seidmann A (2002) The optimal software licensing policy under quality uncertainty. In: Proc 23rd international conference on information systems, Barcelona

Zhang Z, Tan Y, Dey D (2009) Price competition with service level guarantee in web services. Decis Support Syst 47(2):93-104 